\title{
Wolbachia and nuclear-nuclear interactions contribute to reproductive incompatibility in the spider mite Panonychus mori (Acari: Tetranychidae)
}

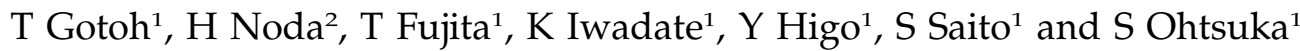 \\ ${ }^{1}$ Faculty of Agriculture, Ibaraki University, Ami, Ibaraki 300-0393, Japan; ${ }^{2}$ National Institute of Agrobiological Sciences, Tsukuba, \\ Ibaraki 305-8634, Japan
}

\begin{abstract}
Maternally transmitted bacteria of the genus Wolbachia are obligate, intracellular symbionts that are responsible for cytoplasmic incompatibility in a wide range of arthropods such as insects and mites. Spider mites often show uni- and bidirectional incompatibilities among populations with and without Wolbachia. Therefore, we surveyed the presence of Wolbachia by PCR and then conducted crossing experiments among 25 populations of Panonychus mori to determine how Wolbachia are related to the incompatibility in this species. Five out of the 25 populations were infected with Wolbachia. These five populations were treated with an antibiotic (rifampicin) to eliminate Wolbachia. We carried out round-robin crosses among 20 Wolbachia-uninfected populations, five infected populations and five rifampicin-treated populations $(30 \times 30=900$ crosses in total). Incompatibility among $P$. mori populations was caused by Wolbachia
\end{abstract}

infection, nuclear-cytoplasmic interactions or nuclear-nuclear interactions. Wolbachia-mediated incompatibility was observed in crosses between uninfected females and infected males or between females and males harboring different Wolbachia strains. Nuclear-cytoplasmic interactions may be responsible for the unidirectional incompatibility in crosses between the two northern populations and one of the southern populations. Bidirectional incompatibility caused by nuclear-nuclear interactions was observed in 99 combinations of interpopulation crosses $(99 / 300=0.33)$. Although no geographical trends were detected in the distribution of bidirectionally compatible populations, the results reveal a genetic divergence among $P$. mori populations.

Heredity (2005) 94, 237-246. doi:10.1038/sj.hdy.6800605

Published online 1 December 2004

Keywords: Wolbachia; Panonychus mori; cytoplasmic incompatibility; reproductive incompatibility; spider mite

\section{Introduction}

Intracellular bacteria of the genus Wolbachia are the causative agents of cytoplasmic incompatibility (CI) found in many arthropod species such as insects (Werren and O'Neill, 1997) and mites (Breeuwer, 1997). Wolbachiainduced CI results in embryonic lethality and/or a malebiased sex ratio in crosses between males that are infected with Wolbachia and females that are uninfected or infected with a different Wolbachia strain (O'Neill et al, 1997). CI results in the production of few or no offspring in diplodiploids (Hoffmann et al, 1986) or in the production of only male offspring in haplodiploids (Breeuwer and Werren, 1990). The CI mechanism has been explained by the modification/rescue model: Wolbachia modify sperm or male chromosomes in the host (mod + ; modification capable) and this modification, which is normally detrimental to the host, is rescued by the same kind of Wolbachia in the females (resc + ; rescue capable) (O'Neill et al, 1997; Bourtzis et al, 1998). Some Wolbachia strains (mod- resc + ) do not

Correspondence: T Gotoh, Laboratory of Applied Entomology and Zoology, Faculty of Agriculture, Ibaraki University, Ami, Ibaraki 300-0393, Japan. E-mail: gotoh@mx.ibaraki.ac.jp

Received 4 November 2003; accepted 13 September 2004; published online 1 December 2004 modify the sperm but rescue the detrimental modification caused by a mod + resc + strain (Bourtzis et al, 1998; Mercot and Poinsot, 1998).

Antibiotics, such as tetracycline and rifampicin, eliminate Wolbachia from the host (O'Neill, 1989; Stouthamer et al, 1990; Noda et al, 2001). When insects are infected with CI-inducing Wolbachia, the crosses between antibiotic-treated females and untreated males are incompatible, but the reciprocal crosses are compatible. Antibiotics can also eliminate Wolbachia from spider mites (Breeuwer, 1997; Gomi et al, 1997; Gotoh et al, 2003).

The CI of the spider mites Tetranychus turkestani and T. urticae (red-form) was found through crossing experiments between antibiotic-treated females and untreated males. The CI observed in these spider mite species was shown by the death of fertilized eggs (Breeuwer, 1997), although incompatibility in haplodiploids was expected to produce all male offspring or a male-biased sex ratio without any death of eggs. On the other hand, Wolbachia infection does not induce reproductive incompatibility in T. kanzawai (Gomi et al, 1997; Gotoh et al, 1999a) or T. urticae (green-form) (Gotoh et al, 1999b), although many populations collected from a wide range of Japanese islands were tested from these species. The present study is of another spider mite species, Panonychus mori, which showed bidirectional reproductive incompatibility in crosses between the southwestern 
population at Tottori $\left(35^{\circ} \mathrm{N}\right)$ and the northern population at Sapporo $\left(43^{\circ} \mathrm{N}\right)$ and resulted in an abnormal malebiased sex ratio (ca. 97-99\% males) (Gotoh and Higo, 1997). Bidirectional incompatibility is assumed to be caused by either nuclear-nuclear interactions, which are the result of lethal combinations of genes, or infection by different Wolbachia strains. Our preliminary crossing experiments for this species also indicated that the crosses between females of some populations and males of other populations are incompatible, but the reciprocal crosses are compatible and give a female-biased sex ratio. This unidirectional incompatibility may be due to infection by Wolbachia.

In the present study, we surveyed for the presence of Wolbachia by PCR and conducted crossing experiments to determine the relationship between Wolbachia infection and reproductive incompatibility observed among the populations of $P$. mori. We also analyzed the possible causes of the reproductive incompatibility observed among Wolbachia-free populations that were prepared by treating the Wolbachia-infected populations with an antibiotic, as well as Wolbachia-uninfected populations of P. mori. We then carried out round-robin crosses among Wolbachia-uninfected populations, Wolbachia-infected populations and Wolbachia-free populations.

\section{Materials and methods}

\section{Mite populations}

Mites were collected from mulberry, Morus bombycis, at 25 localities from 1993 to 1998 (Figure 1). Laboratory stocks of these populations were separately reared on leaf discs (ca. $25 \mathrm{~cm}^{2}$ ) of mulberry placed on watersaturated polyurethane mats in plastic dishes $(9 \mathrm{~cm}$ in diameter). In winter, mulberry plants were cultivated in a greenhouse. All experiments were carried out at $25^{\circ} \mathrm{C}$, $60-70 \% \mathrm{RH}$ and with a $16 \mathrm{~L}-8 \mathrm{D}$ photoperiod.

\section{DNA extraction and PCR amplification}

The PCR techniques used here were the same as those used by Gomi et al (1997) and Gotoh et al (2003). Two pairs of Wolbachia-specific primers were used to detect the presence of Wolbachia. One amplifies a part of the $16 \mathrm{~S}$ rDNA (O'Neill et al, 1992) and the other amplifies the ftsZ gene (Holden et al, 1993). A total of 10-20 females were tested for each population. Since CLO (Cytophaga-like organisms) in the CFB (Cytophaga-Flavobacterium-Bacteroides) phylum have recently been reported to cause reproductive alteration in host insects and mites (Weeks et al, 2001, 2003; Zchori-Fein et al, 2001; Hunter et al, 2003), mite samples were examined using specific $16 S$ rDNA primers (forward 5'-GGAACCTTACCTGGGCTAGAATGTATT and reverse 5'-GAGGGTTCTTTCGGGACGGAAT) based on the 16S rDNA of CLO from Ixodes scapularis (Kurtti et al, 1996), Brevipalpus phoenicis (Weeks et al, 2001) and Encarsia spp. (Zchori-Fein et al, 2001).

\section{Antibiotic treatment}

To administer rifampicin to spider mites, small mulberry leaf discs (ca. $1 \mathrm{~cm}^{2}$ ) were placed on a cotton bed soaked in rifampicin $(0.05 \%, \mathrm{w} / \mathrm{v})$ in plastic dishes $(9 \mathrm{~cm}$ in diameter) 1 day before the start of rearing. Newly hatched larvae were placed on the leaf discs and distilled

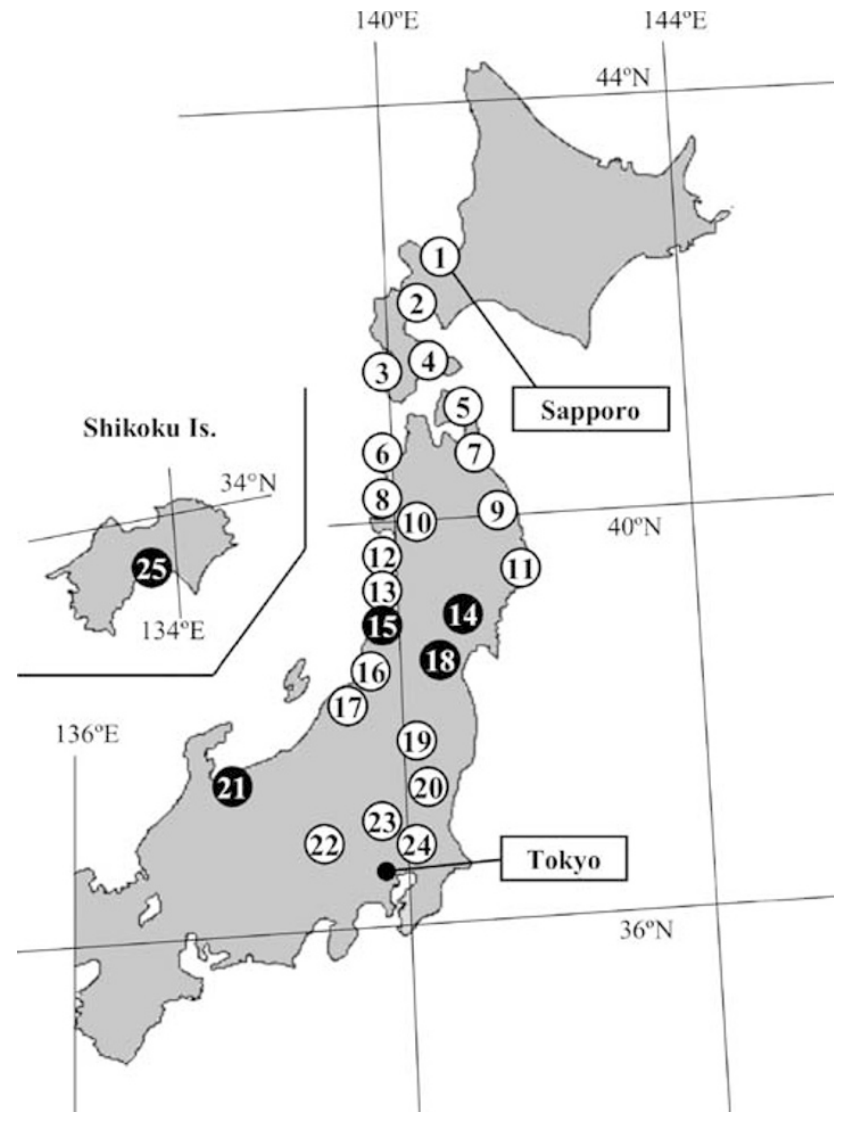

Figure 1 Collection sites and Wolbachia infection in P. mori. Closed and open circles denote Wolbachia-infected and Wolbachia-free populations, respectively.

water was added daily to keep the cotton bed wet. The cotton and the leaf discs were replaced every 4 days (see also Gotoh et al, 1995) and the mites were reared under the antibiotic for one generation. Mites were checked for infection of Wolbachia by PCR after three generations. Offspring from adults that were found to be uninfected were allowed to mate.

\section{Crossing experiments}

In order to determine the reproductive compatibility among the 25 populations, round-robin crosses were carried out on mulberry leaves. Single females in the teleiochrysalis stage from each population were placed on small leaf discs (ca. $4 \mathrm{~cm}^{2}$ ) with a male adult from each of the populations. Crossing experiments were also carried out between the 25 populations and Wolbachiafree populations that were made by treating the Wolbachia-infected populations with rifampicin. The males were removed 2 days after adult emergence of the females. After oviposition started, each female was allowed to lay eggs for 5 days and then removed. Eggs on the leaf discs were checked daily to determine hatchability, survival rate and sex ratio. More than seven pairs were examined for each combination of the crosses and a total of 14480 pairs were used for 900 crosses. Experiments were carried out for 3 years from June to October when good mulberry leaves were available.

Crosses were regarded as compatible when (1) egg hatchability was $\geq 94.1 \%$, (2) survival rate in immatures 
was $\geq 85.0 \%$ and (3) the proportion of female adults was $\geq 60.9 \%$. These values correspond to the minimal rates previously obtained from intrapopulation crosses (Gotoh and Higo, 1997). Data were analyzed with either the Kruskal-Wallis test or ANOVA and the values were tested by the Scheffé's test. Arcsin-transformed values for ANOVA were used for analyzing egg hatchability, survival rate and female ratio.

\section{Results}

\section{Wolbachia infection}

Five out of the 25 populations of $P$. mori were infected with Wolbachia. The primers for a 770-bp fragment of the ftsZ gene and an 890-bp fragment of the 16S rDNA amplified DNAs of the expected sizes in the five populations (Figure 1). These five populations were treated with rifampicin and used for crossing experiments. Hence, using 20 Wolbachia-uninfected populations, five Wolbachia-infected populations and five rifampicin-treated populations, we conducted $30 \times 30=900$ crosses (Figure 2). Detailed results of the crossing experiments are available at http://wwwa. agr.ibaraki.ac.jp/ shokubutu/gotoh/900data.pdf, from supplementary information on Heredity website, or from the author.
Compatibility in intrapopulation crosses of the five Wolbachia-infected populations

Hatchability of eggs, survival rate in immature stages and female ratio were normal, even when infected males of the Sd population were crossed with Wolbachia-free (rifampicin-treated) Sd females (Table 1). This suggests that the Wolbachia strain infecting the Sd population is modification-negative.

On the other hand, the crosses between Wolbachiainfected and rifampicin-treated Wolbachia-free mites in the Hy, Ty, $\mathrm{Hr}$ and Tr populations showed unidirectional incompatibility; that is, crosses between infected males and uninfected females were incompatible, while the reciprocal crosses were compatible (Table 1). The reproductive incompatibility observed in these crosses was detected by a reduced hatchability and/or malebiased sex ratio. This shows that Wolbachia strains in the four $P$. mori populations are modification-positive.

\section{Compatibility among Wolbachia-infected populations}

All the crosses between the Sd and Hy populations were compatible except for the cross between the Sd R females and the Hy males, which was incompatible (top panel in Table 2). In the crosses between the Sd and Ty populations, compatibility was found only in the crosses in which females were mated with uninfected males

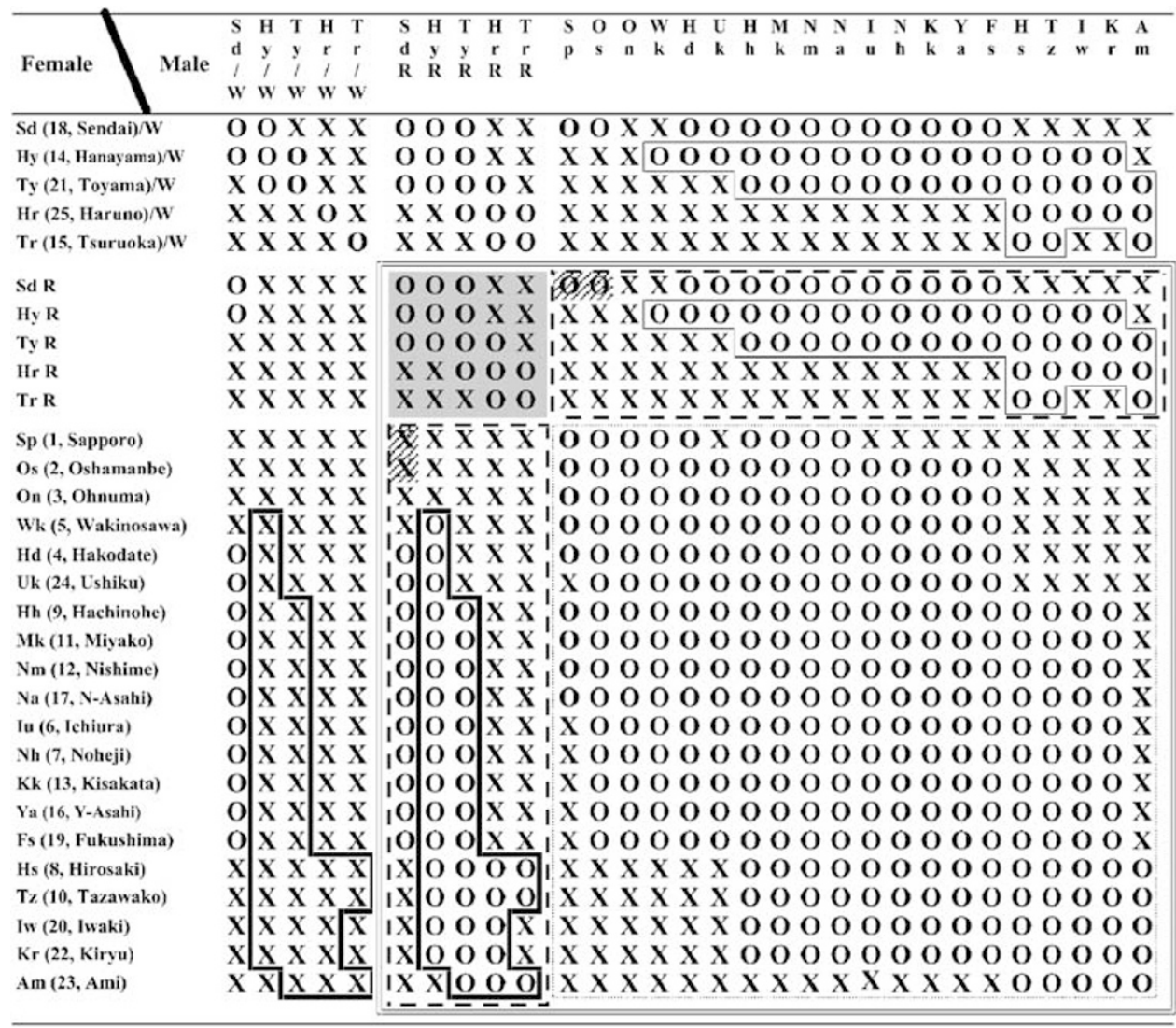

Figure 2 Reproductive compatibility among 25 populations and five rifampicin-treated populations of $P$. mori. Circles show compatible crosses ( $\geq 94.1 \%$ in hatchability of eggs, $\geq 85.0 \%$ in survival rate in immatures and $\geq 60.9 \%$ in female ratio), and Xs show incompatible crosses. W means Wolbachia-infected populations, and R means rifampicin treatment. Localities are shown by abbreviations of two letters. Numbers in parentheses correspond to the site numbers appearing in Figure 1 and full locality names are also shown in parentheses. Crosses enclosed within thick lines indicate Wolbachia-uninfected populations whose reproductive incompatibility is mediated by Wolbachia, and crosses enclosed within thin lines indicate the respective reciprocal crosses of the crosses enclosed within thick lines. 
Table 1 Compatibility of intrapopulation crosses for the five Wolbachia-infected populations of P. mori

\begin{tabular}{|c|c|c|c|c|c|c|}
\hline Cross & $\mathrm{n}^{\mathrm{a}}$ & No. of eggs/female & Hatchability (\%) & Survival rate in immature stages (\%) & $\%$ Female offspring & Compatibility ${ }^{\mathrm{b}}$ \\
\hline \multicolumn{7}{|l|}{ Female $\times$ male } \\
\hline $\mathrm{Sd} \times \mathrm{Sd}$ & 20 & $32.4 \pm 0.74 \mathrm{a}$ & $99.2 \pm 0.32 b$ & $98.7 \pm 0.46$ & $79.0 \pm 0.60 \mathrm{c}$ & $\mathrm{O}$ \\
\hline $\mathrm{Sd} \times \mathrm{Sd} \mathrm{R}$ & 18 & $41.6 \pm 2.03 \mathrm{~b}$ & $97.5 \pm 0.24 \mathrm{ab}$ & $98.6 \pm 0.32$ & $71.8 \pm 1.37 \mathrm{~b}$ & $\mathrm{O}$ \\
\hline $\mathrm{Sd} \mathrm{R} \times \mathrm{Sd}$ & 30 & $32.9 \pm 1.07 \mathrm{a}$ & $96.5 \pm 0.44 \mathrm{a}$ & $98.3 \pm 0.40$ & $66.8 \pm 1.01 \mathrm{a}$ & $\mathrm{O}$ \\
\hline $\mathrm{Sd} R \times \mathrm{Sd} R$ & 15 & $32.4 \pm 1.60 \mathrm{a}$ & $96.7 \pm 0.64 \mathrm{a}$ & $98.9 \pm 0.40$ & $77.4 \pm 1.46 \mathrm{c}$ & $\mathrm{O}$ \\
\hline$\chi^{2}$-value ${ }^{c}$ & & $16.429^{* * *}$ & $20.273^{* * *}$ & $1.069 \mathrm{NS}$ & $42.847^{* * *}$ & \\
\hline $\mathrm{Hy} \times \mathrm{Hy}$ & 15 & $34.9 \pm 2.46 b$ & $97.5 \pm 0.78 b$ & $97.6 \pm 0.49$ & $83.1 \pm 1.29 \mathrm{~d}$ & $\mathrm{O}$ \\
\hline Hy $\times$ Hy R & 17 & $30.1 \pm 1.82 \mathrm{ab}$ & $97.7 \pm 0.58 \mathrm{~b}$ & $97.1 \pm 0.54$ & $64.9 \pm 1.56 \mathrm{~b}$ & $\mathrm{O}$ \\
\hline Hy $\mathrm{R} \times \mathrm{Hy}$ & 15 & $26.1 \pm 1.44 \mathrm{a}$ & $83.8 \pm 2.33 a$ & $95.9 \pm 1.04$ & $36.3 \pm 2.28 \mathrm{a}$ & $X$ \\
\hline Hy R $\times$ Hy R & 19 & $35.1 \pm 1.57 \mathrm{~b}$ & $97.6 \pm 0.44 b$ & $98.5 \pm 0.38$ & $75.8 \pm 1.37 \mathrm{c}$ & $\mathrm{O}$ \\
\hline$\chi^{2}$-value & & $15.031^{* *}$ & $34.497^{* * *}$ & $6.689 \mathrm{NS}$ & $53.685^{* * *}$ & \\
\hline $\mathrm{Ty} \times \mathrm{Ty}$ & 15 & $32.1 \pm 0.96$ & $97.9 \pm 0.50$ & $97.8 \pm 0.63$ & $81.6 \pm 1.26 \mathrm{c}$ & $\mathrm{O}$ \\
\hline Ty $\times$ Ty R & 24 & $32.6 \pm 1.40$ & $96.8 \pm 0.52$ & $96.5 \pm 0.68$ & $71.9 \pm 1.03 \mathrm{~b}$ & $\mathrm{O}$ \\
\hline Ty $R \times$ Ty & 15 & $30.3 \pm 1.60$ & $96.5 \pm 0.35$ & $98.8 \pm 0.59$ & $25.5 \pm 1.39 \mathrm{a}$ & $X$ \\
\hline Ty $R \times$ Ty $R$ & 24 & $34.0 \pm 1.28$ & $97.7 \pm 0.59$ & $97.8 \pm 0.51$ & $72.7 \pm 1.08 \mathrm{~b}$ & $\mathrm{O}$ \\
\hline$\chi^{2}$-value & & $5.508 \mathrm{NS}$ & 5.412 NS & $6.511 \mathrm{NS}$ & $50.842^{* * *}$ & \\
\hline $\mathrm{Hr} \times \mathrm{Hr}$ & 20 & $32.6 \pm 1.06$ & $96.1 \pm 0.64$ & $97.0 \pm 0.71$ & $70.9 \pm 1.33 \mathrm{~b}$ & $\mathrm{O}$ \\
\hline $\mathrm{Hr} \times \mathrm{Hr} \mathrm{R}$ & 18 & $33.9 \pm 1.58$ & $96.4 \pm 0.57$ & $98.4 \pm 0.41$ & $66.1 \pm 1.01 \mathrm{~b}$ & $\mathrm{O}$ \\
\hline $\mathrm{Hr} \mathrm{R} \times \mathrm{Hr}$ & 15 & $35.5 \pm 1.42$ & $97.3 \pm 0.75$ & $98.2 \pm 0.40$ & $27.2 \pm 2.80 \mathrm{a}$ & $x$ \\
\hline $\operatorname{Hr} \mathrm{R} \times \operatorname{Hr} \mathrm{R}$ & 14 & $34.8 \pm 1.91$ & $98.2 \pm 0.44$ & $99.3 \pm 0.40$ & $70.4 \pm 1.87 \mathrm{~b}$ & $\mathrm{O}$ \\
\hline$\chi^{2}$-value & & $2.433 \mathrm{NS}$ & $5.160 \mathrm{NS}$ & $5.699 \mathrm{NS}$ & $38.410^{* * *}$ & \\
\hline $\operatorname{Tr} \times \operatorname{Tr}$ & 14 & $41.0 \pm 2.31 \mathrm{~b}$ & $96.7 \pm 0.87$ & $93.7 \pm 0.90 \mathrm{a}$ & $73.6 \pm 1.76 \mathrm{~b}$ & $\mathrm{O}$ \\
\hline $\operatorname{Tr} \times \operatorname{Tr} \mathrm{R}$ & 16 & $32.5 \pm 1.72 \mathrm{a}$ & $96.2 \pm 0.64$ & $98.7 \pm 0.45 b$ & $73.6 \pm 1.23 \mathrm{~b}$ & $\mathrm{O}$ \\
\hline $\operatorname{Tr} \mathrm{R} \times \operatorname{Tr}$ & 17 & $30.5 \pm 1.50 \mathrm{a}$ & $96.3 \pm 0.72$ & $99.2 \pm 0.38 \mathrm{~b}$ & $16.4 \pm 1.41 \mathrm{a}$ & $x$ \\
\hline $\operatorname{Tr} \mathrm{R} \times \operatorname{Tr} \mathrm{R}$ & 17 & $30.7 \pm 1.18 \mathrm{a}$ & $96.7 \pm 0.47$ & $99.8 \pm 0.19 \mathrm{~b}$ & $77.3 \pm 1.47 \mathrm{~b}$ & $\mathrm{O}$ \\
\hline$\chi^{2}$-value & & $12.362^{* *}$ & $0.453 \mathrm{NS}$ & $33.717^{* * *}$ & $39.290^{* * *}$ & \\
\hline
\end{tabular}

aNumber of females tested.

${ }^{\mathrm{b} C}$ Circles show compatible crosses and Xs incompatible ones.

cMeans differ significantly at $P<0.01$ (**) $^{* *}$ and $P<0.001$ (***) (Kruskal-Wallis test); NS, not significant at $P>0.05$. Data are shown as mean \pm SE.

Values in a column followed by different letters are significantly different at $P<0.05$ (Scheffé's test).

(middle panel in Table 2). The Hy and Ty populations were infected with the same strain of Wolbachia (bottom panel in Table 2), as shown by the crosses between infected males and infected females being compatible, while it is difficult to explain the properties the Wolbachia strain infecting the Sd population. The Sd population seems to harbor a modification-negative $(\bmod -)$ strain of Wolbachia based on its crosses with the Hy populations, while it seems to harbor a modification-positive $(\bmod +)$ strain based on its crosses with the Ty population. Thus, these results seem to be contradictory.

Crosses between infected mites from the $\mathrm{Hr}$ and $\mathrm{Tr}$ populations were incompatible, but those between uninfected males and either infected or uninfected females were compatible (Table 3). This shows that the $\mathrm{Hr}$ and $\mathrm{Tr}$ populations were infected with different Wolbachia strains.

Table 4 shows the results of the crosses among the Sd, $\mathrm{Hy}, \mathrm{Hr}$ and $\mathrm{Tr}$ populations. All the crosses between the Sd population and either the $\mathrm{Hr}$ or $\mathrm{Tr}$ population were incompatible and female ratios were significantly lower $\left(\mathrm{Sd} \times \mathrm{Hr}: \quad\right.$ ANOVA $, \quad \mathrm{F}_{9,157}=81.327, \quad P<0.001 ; \quad \mathrm{Sd} \times \mathrm{Tr}$ : ANOVA, $\left.F_{9,154}=154.960, P<0.001\right)$ than those in the intrapopulation crosses. Incompatibility was also ob- served even when rifampicin-treated individuals were crossed. Similarly, the crosses between the Hy population and either the $\mathrm{Hr}$ or $\mathrm{Tr}$ population were incompatible (Table 4). The female ratios were significantly lower $\left(\mathrm{Hy} \times \mathrm{Hr}\right.$ : ANOVA, $\mathrm{F}_{9,148}=94.923, P<0.001 ; \mathrm{Hy} \times \mathrm{Tr}$ : ANOVA, $\mathrm{F}_{9,162}=85.145, P<0.001$ ) than those in each intrapopulation cross. These results imply that the incompatibility observed in crosses between either the Sd or Hy population and either the Hr or Tr population is due to nuclear-nuclear interactions rather than to the microorganisms.

The Ty and Hr populations harbored different Wolbachia strains (Table 5), because the crosses between infected males and infected females from these two populations were incompatible but the crosses between Wolbachia-free males and either of Wolbachia-infected females or Wolbachia-free females were compatible. In contrast, none of the crosses between the Ty and $\mathrm{Tr}$ populations were compatible (Table 5), implying that the incompatibility between them is caused by nuclear factors. The female ratios in the crosses between Ty and $\operatorname{Tr}$ populations were significantly lower (ANOVA, $\left.\mathrm{F}_{9,190}=83.539, P<0.001\right)$ than those in the intrapopulation crosses. 
Table 2 Compatibility among three Wolbachia-infected populations of P. mori (Sd, Hy and Ty)

\begin{tabular}{|c|c|c|c|c|c|c|}
\hline Cross & $\mathrm{n}^{\mathrm{a}}$ & No. of eggs/female & Hatchability (\%) & Survival rate in immature stages (\%) & $\%$ Female offspring & Compatibility $^{\mathrm{b}}$ \\
\hline \multicolumn{7}{|l|}{ Female $\times$ male } \\
\hline $\mathrm{Sd} \times \mathrm{Hy}$ & 23 & $25.8 \pm 1.48 \mathrm{a}$ & $98.7 \pm 0.50 \mathrm{~b}$ & $93.7 \pm 1.04$ & $76.2 \pm 0.77 \mathrm{c}$ & $\mathrm{O}$ \\
\hline $\mathrm{Sd} \times \mathrm{Hy} \mathrm{R}$ & 15 & $25.2 \pm 0.92 \mathrm{a}$ & $96.9 \pm 0.67 \mathrm{ab}$ & $96.3 \pm 0.95$ & $66.6 \pm 1.53 \mathrm{~b}$ & $\mathrm{O}$ \\
\hline $\mathrm{Sd} \mathrm{R} \times \mathrm{Hy}$ & 15 & $30.9 \pm 2.18 \mathrm{a}$ & $91.6 \pm 2.93 \mathrm{a}$ & $96.9 \pm 0.88$ & $36.6 \pm 2.57 \mathrm{a}$ & $X$ \\
\hline Sd R $\times$ Hy R & 16 & $26.1 \pm 1.46 \mathrm{a}$ & $98.1 \pm 0.59 \mathrm{ab}$ & $97.0 \pm 0.69$ & $67.4 \pm 1.50 \mathrm{bc}$ & $\mathrm{O}$ \\
\hline $\mathrm{Hy} \times \mathrm{Sd}(1)^{\mathrm{c}}$ & 16 & $31.9 \pm 1.44 \mathrm{a}$ & $97.6 \pm 0.73 \mathrm{ab}$ & $96.3 \pm 0.64$ & $65.0 \pm 1.30 \mathrm{~b}$ & $\mathrm{O}$ \\
\hline Hy × Sd (2) & 14 & $30.2 \pm 1.85 \mathrm{a}$ & $96.8 \pm 0.68 \mathrm{ab}$ & $93.2 \pm 1.36$ & $71.9 \pm 1.21 \mathrm{bc}$ & $\mathrm{O}$ \\
\hline $\mathrm{Hy} \times \mathrm{Sd} \mathrm{R}$ & 17 & $26.4 \pm 1.42 \mathrm{a}$ & $98.0 \pm 0.64 \mathrm{ab}$ & $97.0 \pm 0.61$ & $69.7 \pm 1.30 \mathrm{bc}$ & $\mathrm{O}$ \\
\hline Hy $\mathrm{R} \times \mathrm{Sd}(1)$ & 12 & $23.6 \pm 0.65 \mathrm{a}$ & $95.8 \pm 1.19 \mathrm{ab}$ & $96.8 \pm 1.04$ & $63.9 \pm 1.71 \mathrm{~b}$ & $\mathrm{O}$ \\
\hline Hy $\mathrm{R} \times \mathrm{Sd}(2)$ & 11 & $23.4 \pm 1.20 \mathrm{a}$ & $97.2 \pm 0.69 \mathrm{ab}$ & $94.1 \pm 1.45$ & $67.3 \pm 1.79 b c$ & $\mathrm{O}$ \\
\hline Hy $\mathrm{R} \times \mathrm{Sd} \mathrm{R}$ & 14 & $22.9 \pm 0.93$ a & $98.7 \pm 0.59 \mathrm{~b}$ & $96.7 \pm 0.73$ & $68.5 \pm 1.49 \mathrm{bc}$ & $\mathrm{O}$ \\
\hline$\chi^{2}$-value ${ }^{\mathrm{d}}$ & & $33.186^{* * *}$ & $18.525^{*}$ & $15.687 \mathrm{NS}$ & $82.916^{* * *}$ & \\
\hline $\mathrm{Sd} \times \mathrm{Ty}(1)$ & 25 & $32.6 \pm 0.59 \mathrm{ab}$ & $98.7 \pm 0.34 b$ & $98.1 \pm 0.45 b c$ & $7.3 \pm 0.97 \mathrm{a}$ & $X$ \\
\hline Sd × Ty (2) & 22 & $35.0 \pm 1.71 \mathrm{~b}$ & $96.8 \pm 0.30 \mathrm{~b}$ & $99.6 \pm 0.27 \mathrm{c}$ & $22.9 \pm 1.70 \mathrm{~b}$ & $X$ \\
\hline $\mathrm{Sd} \times \mathrm{Ty} \mathrm{R}$ & 16 & $30.8 \pm 1.68 \mathrm{ab}$ & $95.8 \pm 0.57 \mathrm{~b}$ & $99.5 \pm 0.29 \mathrm{c}$ & $65.6 \pm 1.84 \mathrm{~d}$ & $\mathrm{O}$ \\
\hline $\mathrm{Sd} R \times \mathrm{Ty}$ & 19 & $33.2 \pm 1.78 \mathrm{ab}$ & $98.5 \pm 0.50 \mathrm{~b}$ & $99.4 \pm 0.35 \mathrm{c}$ & $36.6 \pm 2.37 c$ & $X$ \\
\hline Sd R $\times$ Ty R & 15 & $30.2 \pm 1.36 \mathrm{ab}$ & $96.7 \pm 0.60 \mathrm{~b}$ & $99.1 \pm 0.39 c$ & $71.3 \pm 1.66 \mathrm{~d}$ & $\mathrm{O}$ \\
\hline Ty × Sd (1) & 15 & $32.9 \pm 0.87 \mathrm{ab}$ & $99.2 \pm 0.35 b$ & $97.5 \pm 0.61 \mathrm{bc}$ & $20.1 \pm 1.48 \mathrm{ab}$ & $X$ \\
\hline Ty $\times$ Sd (2) & 19 & $30.8 \pm 1.09 \mathrm{ab}$ & $96.9 \pm 0.56 \mathrm{~b}$ & $99.3 \pm 0.33 c$ & $29.1 \pm 2.39 b c$ & $X$ \\
\hline Ty $\times$ Sd (3) & 16 & $27.9 \pm 1.38 \mathrm{ab}$ & $83.4 \pm 2.35 \mathrm{a}$ & $94.3 \pm 1.18 \mathrm{ab}$ & $30.3 \pm 3.48 \mathrm{bc}$ & $X$ \\
\hline Ty $\times$ Sd R & 17 & $34.4 \pm 1.68 \mathrm{ab}$ & $96.9 \pm 0.50 \mathrm{~b}$ & $98.2 \pm 0.46 \mathrm{c}$ & $71.3 \pm 1.39 \mathrm{~d}$ & $\mathrm{O}$ \\
\hline Ty $\mathrm{R} \times \mathrm{Sd}(1)$ & 16 & $31.2 \pm 1.26 \mathrm{ab}$ & $97.2 \pm 0.55 \mathrm{~b}$ & $98.3 \pm 0.81 \mathrm{c}$ & $37.3 \pm 2.98 \mathrm{c}$ & $X$ \\
\hline Ty $\mathrm{R} \times \mathrm{Sd}(2)$ & 12 & $25.3 \pm 1.50 \mathrm{a}$ & $77.9 \pm 4.90 \mathrm{a}$ & $93.0 \pm 1.23 \mathrm{a}$ & $40.0 \pm 2.09 \mathrm{c}$ & $X$ \\
\hline Ty $R \times S d R$ & 14 & $30.8 \pm 1.83 \mathrm{ab}$ & $94.8 \pm 1.09 \mathrm{~b}$ & $98.4 \pm 0.47 \mathrm{c}$ & $67.3 \pm 1.48 \mathrm{~d}$ & $\mathrm{O}$ \\
\hline$\chi^{2}$-value & & $29.186^{* *}$ & $85.094^{* * *}$ & $58.336^{* * *}$ & $171.337^{* * *}$ & \\
\hline $\mathrm{Hy} \times \mathrm{Ty}$ & 18 & $30.5 \pm 1.25$ & $96.5 \pm 0.60 \mathrm{~b}$ & $97.9 \pm 0.63 \mathrm{a}$ & $75.8 \pm 1.43 c$ & $\mathrm{O}$ \\
\hline Hy $\times$ Ty R & 18 & $33.2 \pm 1.68$ & $97.3 \pm 0.42 b$ & $97.2 \pm 0.35 \mathrm{a}$ & $78.1 \pm 1.32 \mathrm{c}$ & $\mathrm{O}$ \\
\hline Hy $R \times$ Ty & 15 & $37.2 \pm 1.90$ & $73.6 \pm 4.12 \mathrm{a}$ & $98.5 \pm 0.67 \mathrm{a}$ & $55.6 \pm 2.98 \mathrm{~b}$ & $X$ \\
\hline Hy $R \times$ Ty $R$ & 17 & $33.8 \pm 1.65$ & $97.2 \pm 0.53 b$ & $99.1 \pm 0.35$ a & $71.0 \pm 1.78 \mathrm{c}$ & $\mathrm{O}$ \\
\hline $\mathrm{Ty} \times \mathrm{Hy}$ & 17 & $32.9 \pm 1.41$ & $97.1 \pm 0.47 b$ & $99.3 \pm 0.40 \mathrm{a}$ & $77.7 \pm 1.83 \mathrm{c}$ & $\mathrm{O}$ \\
\hline Ty $\times$ Hy R & 21 & $30.6 \pm 0.89$ & $96.5 \pm 0.61 b$ & $98.3 \pm 0.51 \mathrm{a}$ & $74.1 \pm 1.21 \mathrm{c}$ & $\mathrm{O}$ \\
\hline Ty $\mathrm{R} \times \mathrm{Hy}$ & 15 & $31.5 \pm 2.20$ & $91.0 \pm 3.33 \mathrm{~b}$ & $98.6 \pm 0.47 \mathrm{a}$ & $35.0 \pm 3.62 \mathrm{a}$ & $X$ \\
\hline Ty R $\times$ Hy R & 20 & $30.5 \pm 1.46$ & $96.5 \pm 0.48 b$ & $98.8 \pm 0.38$ a & $74.0 \pm 1.23 \mathrm{c}$ & $\mathrm{O}$ \\
\hline$\chi^{2}$-value & & 11.303 NS & $34.332^{* * *}$ & $17.296^{*}$ & $73.071^{* * *}$ & \\
\hline
\end{tabular}

aNumber of females tested.

${ }^{b}$ Circles show compatible crosses and Xs incompatible ones.

cNumerals in parentheses indicate the number of trials.

${ }^{\mathrm{d}}$ Means differ significantly at $P<0.05\left({ }^{*}\right), P<0.01\left({ }^{* *}\right)$ and $P<0.001(* * *)$ (Kruskal-Wallis test); NS, not significant at $P>0.05$.

Data are shown as mean \pm SE. Values in a column followed by different letters are significantly different at $P<0.05$ (Scheffé's test).

Table 3 Compatibility between the Wolbachia-infected $\mathrm{Hr}$ and $\mathrm{Tr}$ populations of P. mori

\begin{tabular}{|c|c|c|c|c|c|c|}
\hline Cross & $\mathrm{n}^{\mathrm{a}}$ & No. of eggs/female & Hatchability (\%) & Survival rate in immature stages (\%) & $\%$ Female offspring & Compatibility $^{\mathrm{b}}$ \\
\hline \multicolumn{7}{|l|}{ Female $\times$ male } \\
\hline $\mathrm{Hr} \times \operatorname{Tr}$ & 14 & $31.2 \pm 1.08 \mathrm{a}$ & $94.5 \pm 0.94$ & $97.8 \pm 0.71 \mathrm{a}$ & $35.1 \pm 2.02 \mathrm{~b}$ & $x$ \\
\hline $\mathrm{Tr} \times \mathrm{Hr}$ & 18 & $30.1 \pm 1.17 \mathrm{a}$ & $96.7 \pm 0.61$ & $97.3 \pm 0.55 a$ & $19.1 \pm 1.83$ a & $X$ \\
\hline $\mathrm{Hr} \times \operatorname{Tr} \mathrm{R}$ & 19 & $33.2 \pm 1.51 \mathrm{a}$ & $97.7 \pm 0.54$ & $98.3 \pm 0.58 \mathrm{a}$ & $77.4 \pm 1.52 \mathrm{c}$ & $\mathrm{O}$ \\
\hline $\operatorname{Tr} \times \operatorname{Hr} \mathrm{R}$ & 18 & $27.1 \pm 1.28 \mathrm{a}$ & $97.5 \pm 0.54$ & $98.3 \pm 0.46 \mathrm{a}$ & $72.6 \pm 1.30 \mathrm{c}$ & $\mathrm{O}$ \\
\hline $\mathrm{Hr} \mathrm{R} \times \mathrm{Tr}$ & 18 & $32.1 \pm 1.38 \mathrm{a}$ & $97.6 \pm 0.52$ & $99.5 \pm 0.30 \mathrm{a}$ & $18.6 \pm 2.05 \mathrm{a}$ & $X$ \\
\hline $\mathrm{Tr} \mathrm{R} \times \mathrm{Hr}$ & 21 & $28.6 \pm 1.17 \mathrm{a}$ & $97.6 \pm 0.46$ & $98.9 \pm 0.52 \mathrm{a}$ & $14.8 \pm 1.61 \mathrm{a}$ & $X$ \\
\hline Hr R $\times \operatorname{Tr} R$ & 17 & $31.3 \pm 2.03 \mathrm{a}$ & $96.7 \pm 0.50$ & $99.2 \pm 0.35 \mathrm{a}$ & $75.4 \pm 1.42 \mathrm{c}$ & $\mathrm{O}$ \\
\hline $\operatorname{Tr} \mathrm{R} \times \mathrm{Hr} \mathrm{R}$ & 19 & $30.8 \pm 0.97$ a & $96.6 \pm 0.30$ & $98.2 \pm 0.41$ a & $70.4 \pm 1.77 \mathrm{c}$ & $\mathrm{O}$ \\
\hline$\chi^{2}$-value ${ }^{c}$ & & $12.700^{*}$ & 10.739 NS & $14.830^{*}$ & $101.448^{* * *}$ & \\
\hline
\end{tabular}

aNumber of females tested.

${ }^{\mathrm{b}}$ Circles show compatible crosses and Xs incompatible ones.

'Means differ significantly at $P<0.05\left(^{*}\right)$ and $P<0.001{ }^{(* * *}$ (Kruskal-Wallis test); NS, not significant at $P>0.05$. Data are shown as mean \pm SE.

Values in a column followed by different letters are significantly different at $P<0.05$ (Scheffé's test). 
Table 4 Compatibility of crosses between either of the Sd or Hy populations and either of the Hr or Tr populations of $P$. mori

\begin{tabular}{|c|c|c|c|c|c|c|}
\hline Cross & $\mathrm{n}^{\mathrm{a}}$ & No. of eggs/female & Hatchability (\%) & Survival rate in immature stages (\%) & $\%$ Female offspring & Compatibility ${ }^{\mathrm{b}}$ \\
\hline \multicolumn{7}{|l|}{ Female $\times$ male } \\
\hline $\mathrm{Sd} \times \mathrm{Hr}$ & 15 & $28.8 \pm 1.69 \mathrm{a}$ & $95.9 \pm 0.87 \mathrm{a}$ & $99.4 \pm 0.38 c$ & $15.8 \pm 1.92 \mathrm{ab}$ & $x$ \\
\hline $\mathrm{Hr} \times \mathrm{Sd}$ & 16 & $31.3 \pm 1.68 \mathrm{a}$ & $95.7 \pm 0.68 \mathrm{a}$ & $99.0 \pm 0.40 \mathrm{c}$ & $26.6 \pm 2.42 \mathrm{bcd}$ & $X$ \\
\hline $\mathrm{Sd} \times \mathrm{Hr} \mathrm{R}$ & 15 & $24.7 \pm 1.53 \mathrm{a}$ & $96.6 \pm 0.79 a$ & $94.4 \pm 1.06 \mathrm{ab}$ & $12.8 \pm 2.13 \mathrm{ab}$ & $X$ \\
\hline $\mathrm{Hr} \times \mathrm{Sd} \mathrm{R}$ & 17 & $26.4 \pm 1.76 \mathrm{a}$ & $89.6 \pm 2.87 \mathrm{a}$ & $96.7 \pm 0.61 \mathrm{bc}$ & $37.1 \pm 3.85 \mathrm{~d}$ & $x$ \\
\hline $\mathrm{Sd} \mathrm{R} \times \mathrm{Hr}$ & 14 & $26.5 \pm 1.44 \mathrm{a}$ & $96.1 \pm 1.10 \mathrm{a}$ & $96.1 \pm 0.71 \mathrm{abc}$ & $10.5 \pm 2.79 \mathrm{a}$ & $x$ \\
\hline $\mathrm{Hr} \mathrm{R} \times \mathrm{Sd}$ & 17 & $24.5 \pm 0.73 \mathrm{a}$ & $92.8 \pm 2.13 \mathrm{a}$ & $94.0 \pm 1.05 \mathrm{ab}$ & $21.6 \pm 1.87 \mathrm{abc}$ & $x$ \\
\hline Sd R × Hr R & 15 & $23.9 \pm 0.80 \mathrm{a}$ & $96.4 \pm 0.81 \mathrm{a}$ & $96.7 \pm 0.92 \mathrm{bc}$ & $21.3 \pm 2.84 \mathrm{abc}$ & $X$ \\
\hline $\mathrm{Hr} \mathrm{R} \times \mathrm{Sd} \mathrm{R}$ & 18 & $25.3 \pm 1.15$ a & $92.6 \pm 1.70 \mathrm{a}$ & $92.1 \pm 0.87$ a & $30.6 \pm 2.73 \mathrm{~cd}$ & $x$ \\
\hline$\chi^{2}$-value & & $18.806^{* *}$ & $14.540^{*}$ & $47.037^{* * *}$ & $50.372^{* * *}$ & \\
\hline $\mathrm{Sd} \times \operatorname{Tr}$ & 15 & $35.1 \pm 2.36$ & $96.7 \pm 0.82$ & $98.0 \pm 0.61$ & $38.4 \pm 2.30 \mathrm{c}$ & $x$ \\
\hline $\operatorname{Tr} \times \mathrm{Sd}$ & 18 & $32.4 \pm 1.42$ & $97.3 \pm 0.76$ & $97.9 \pm 0.64$ & $13.2 \pm 1.44 \mathrm{a}$ & $X$ \\
\hline $\mathrm{Sd} \times \operatorname{Tr} \mathrm{R}$ & 18 & $34.4 \pm 1.74$ & $96.6 \pm 0.64$ & $99.1 \pm 0.37$ & $37.1 \pm 2.78 \mathrm{c}$ & $X$ \\
\hline $\operatorname{Tr} \times S d R$ & 16 & $29.3 \pm 1.11$ & $96.6 \pm 0.89$ & $98.6 \pm 0.47$ & $10.4 \pm 1.13 \mathrm{a}$ & $X$ \\
\hline $\mathrm{Sd} \mathrm{R} \times \mathrm{Tr}$ & 15 & $31.1 \pm 1.69$ & $95.2 \pm 0.73$ & $97.9 \pm 0.57$ & $18.1 \pm 1.81 \mathrm{ab}$ & $x$ \\
\hline $\operatorname{Tr} \mathrm{R} \times \mathrm{Sd}$ & 16 & $32.3 \pm 1.07$ & $96.8 \pm 0.51$ & $97.9 \pm 0.50$ & $14.4 \pm 1.59 \mathrm{a}$ & $X$ \\
\hline $\mathrm{Sd} R \times \operatorname{Tr} \mathrm{R}$ & 15 & $30.7 \pm 0.88$ & $96.2 \pm 0.36$ & $99.6 \pm 0.28$ & $28.1 \pm 2.78$ bc & $X$ \\
\hline $\operatorname{Tr} \mathrm{R} \times \mathrm{Sd} \mathrm{R}$ & 17 & $30.2 \pm 1.17$ & $95.2 \pm 0.81$ & $99.0 \pm 0.40$ & $11.3 \pm 0.97 \mathrm{a}$ & $x$ \\
\hline$\chi^{2}$-value & & 9.928 NS & 11.158 NS & 11.138 NS & $80.320^{* * *}$ & \\
\hline $\mathrm{Hy} \times \mathrm{Hr}$ & 16 & $27.7 \pm 1.61 \mathrm{a}$ & $92.2 \pm 1.83 b$ & $96.2 \pm 0.95$ & $2.7 \pm 1.37 \mathrm{a}$ & $x$ \\
\hline $\mathrm{Hr} \times \mathrm{Hy}$ & 15 & $27.4 \pm 1.68 \mathrm{a}$ & $67.9 \pm 4.04 \mathrm{a}$ & $95.7 \pm 1.28$ & $21.6 \pm 3.95 b$ & $x$ \\
\hline $\mathrm{Hy} \times \mathrm{Hr} \mathrm{R}$ & 13 & $24.8 \pm 1.44 \mathrm{a}$ & $96.2 \pm 1.19 \mathrm{~b}$ & $94.6 \pm 1.15$ & $13.4 \pm 2.46 \mathrm{ab}$ & $X$ \\
\hline $\mathrm{Hr} \times$ Hy R & 16 & $31.9 \pm 1.44 \mathrm{a}$ & $94.7 \pm 2.43 \mathrm{~b}$ & $96.5 \pm 0.82$ & $47.3 \pm 2.30 \mathrm{~d}$ & $x$ \\
\hline $\mathrm{Hy} \mathrm{R} \times \mathrm{Hr}$ & 14 & $25.9 \pm 1.40 \mathrm{a}$ & $93.1 \pm 2.92 \mathrm{~b}$ & $95.6 \pm 0.98$ & $6.9 \pm 2.36 \mathrm{a}$ & $X$ \\
\hline $\mathrm{Hr} \mathrm{R} \times \mathrm{Hy}$ & 16 & $24.5 \pm 0.88 \mathrm{a}$ & $88.0 \pm 2.37 \mathrm{~b}$ & $94.6 \pm 1.19$ & $21.2 \pm 1.88 \mathrm{~b}$ & $X$ \\
\hline Hy $R \times$ Hr $R$ & 15 & $28.3 \pm 1.88$ a & $97.5 \pm 0.50 \mathrm{~b}$ & $94.7 \pm 0.91$ & $26.4 \pm 3.00 \mathrm{bc}$ & $x$ \\
\hline Hr R $\times$ Hy R & 18 & $25.8 \pm 0.81$ a & $95.2 \pm 1.03 \mathrm{~b}$ & $95.7 \pm 0.87$ & $37.0 \pm 2.35 \mathrm{~cd}$ & $x$ \\
\hline$\chi^{2}$-value & & $16.820^{*}$ & $40.005^{* * *}$ & $3.898 \mathrm{NS}$ & $83.279^{* * *}$ & \\
\hline $\mathrm{Hy} \times \operatorname{Tr}$ & 17 & $36.2 \pm 2.03$ & $96.5 \pm 0.76 \mathrm{~b}$ & $98.2 \pm 0.35$ & $16.7 \pm 2.11 \mathrm{abc}$ & $x$ \\
\hline $\mathrm{Tr} \times \mathrm{Hy}$ & 20 & $28.6 \pm 1.01$ & $96.6 \pm 0.55 b$ & $98.2 \pm 0.49$ & $21.7 \pm 1.87 \mathrm{abc}$ & $x$ \\
\hline $\mathrm{Hy} \times \operatorname{Tr} \mathrm{R}$ & 18 & $29.4 \pm 1.52$ & $94.4 \pm 1.09 \mathrm{~b}$ & $95.5 \pm 1.04$ & $24.8 \pm 3.28 \mathrm{abc}$ & $X$ \\
\hline $\operatorname{Tr} \times$ Hy R & 20 & $30.4 \pm 1.10$ & $94.9 \pm 1.47 \mathrm{~b}$ & $98.1 \pm 0.46$ & $25.3 \pm 2.89 \mathrm{bc}$ & $x$ \\
\hline Hy $\mathrm{R} \times \mathrm{Tr}$ & 16 & $29.5 \pm 1.97$ & $95.4 \pm 0.84 \mathrm{~b}$ & $98.3 \pm 0.51$ & $12.2 \pm 0.86 \mathrm{a}$ & $X$ \\
\hline $\operatorname{Tr} \mathrm{R} \times \mathrm{Hy}$ & 16 & $29.5 \pm 1.47$ & $96.5 \pm 0.55 b$ & $98.3 \pm 0.50$ & $14.4 \pm 1.36 \mathrm{ab}$ & $X$ \\
\hline Hy $R \times \operatorname{Tr} R$ & 16 & $33.6 \pm 2.01$ & $92.7 \pm 2.08 \mathrm{~b}$ & $98.3 \pm 0.47$ & $20.7 \pm 2.22 \mathrm{abc}$ & $x$ \\
\hline $\operatorname{Tr} \mathrm{R} \times \mathrm{Hy} \mathrm{R}$ & 20 & $30.7 \pm 1.51$ & $76.1 \pm 3.46 \mathrm{a}$ & $99.0 \pm 0.41$ & $27.9 \pm 2.72 \mathrm{c}$ & $x$ \\
\hline$\chi^{2}$-value & & $13.179 \mathrm{NS}$ & $33.338^{* * *}$ & $11.972 \mathrm{NS}$ & $29.326^{* * *}$ & \\
\hline
\end{tabular}

a Number of females tested.

${ }^{\mathrm{b} C}$ Circles show compatible crosses and Xs incompatible ones.

cMeans differ significantly at $P<0.05\left(^{*}\right), P<0.01\left({ }^{* *}\right)$ and $P<0.001\left(^{* * *}\right.$ (Kruskal-Wallis test); NS, not significant at $P>0.05$. Data are shown as mean \pm SE. Values in a column followed by different letters are significantly different at $P<0.05$ (Scheffé's test).

Compatibility among the 30 populations including the five rifampicin-treated populations

Reproductive incompatibility mediated by Wolbachia was found in crosses between 17 Wolbachia-uninfected populations and four infected populations (represented by the region containing Xs enclosed in a thick line in Figure 2). Females from these 17 uninfected populations were incompatible when crossed with males of Wolbachiainfected populations, but they were compatible when crossed with rifampicin-treated males. The reciprocal crosses between females of either Wolbachia-infected or rifampicin-treated populations and males of Wolbachiauninfected populations were compatible (these crosses are shown by circles enclosed by a thin line in Figure 2). As the most typical examples, the crosses between two
Wolbachia-uninfected populations $(\mathrm{Hs}, \mathrm{Tz})$ and four Wolbachia-infected populations ( $\mathrm{Hy}, \mathrm{Ty}, \mathrm{Hr}$ and $\mathrm{Tr}$ ) are shown in Table 6. In haplodiploid species, such as spider mites, incompatibility is usually revealed by male-biased offspring with little or no egg mortality. However, in some crosses, the eggs of P. mori failed to hatch, as was reported in the CI of Tetranychus species (Breeuwer, 1997).

The Wolbachia-infected Sd population was bidirectionally compatible with 11 Wolbachia-uninfected populations (Figure 2), which raises the possibility that this population harbors a modification-negative strain of Wolbachia (but see below). It was considered that the remaining bidirectional incompatibility observed among Wolbachia-uninfected populations (shown by $\mathrm{Xs}_{\mathrm{s}}$ in 
Table 5 Compatibility between the Wolbachia-infected Ty population and either of the $\mathrm{Hr}$ or $\mathrm{Tr}$ populations of $P$. mori

\begin{tabular}{|c|c|c|c|c|c|c|}
\hline Cross & $\mathrm{n}^{\mathrm{a}}$ & No. of eggs/female & Hatchability (\%) & Survival rate in immature stages (\%) & $\%$ Female offspring & Compatibility ${ }^{\mathrm{b}}$ \\
\hline \multicolumn{7}{|l|}{ Female $\times$ male } \\
\hline $\mathrm{Ty} \times \mathrm{Hr}$ & 16 & $30.1 \pm 1.64$ & $96.6 \pm 0.52$ & $97.5 \pm 0.59 a$ & $22.8 \pm 3.05 \mathrm{a}$ & $x$ \\
\hline $\mathrm{Hr} \times \mathrm{Ty}$ & 14 & $33.6 \pm 1.06$ & $95.2 \pm 0.85$ & $98.1 \pm 0.80 \mathrm{a}$ & $25.2 \pm 4.34 \mathrm{a}$ & $X$ \\
\hline $\mathrm{Ty} \times \mathrm{Hr} \mathrm{R}$ & 19 & $29.2+1.09$ & $96.9+0.64$ & $99.3+0.32 \mathrm{a}$ & $70.1+1.21 \mathrm{~b}$ & $\mathrm{O}$ \\
\hline $\mathrm{Hr} \times \mathrm{Ty} \mathrm{R}$ & 18 & $32.0 \pm 1.22$ & $96.5 \pm 0.31$ & $99.5 \pm 0.29 \mathrm{a}$ & $73.4 \pm 2.30 \mathrm{~b}$ & $\mathrm{O}$ \\
\hline Ty $\mathrm{R} \times \mathrm{Hr}$ & 24 & $29.9 \pm 1.01$ & $96.6 \pm 0.36$ & $99.8 \pm 0.17 \mathrm{a}$ & $20.0 \pm 1.94 \mathrm{a}$ & $X$ \\
\hline $\mathrm{Hr} \mathrm{R} \times \mathrm{Ty}$ & 20 & $32.9 \pm 2.03$ & $97.1 \pm 0.92$ & $98.9 \pm 0.38 \mathrm{a}$ & $17.4 \pm 2.60 \mathrm{a}$ & $\mathrm{X}$ \\
\hline Ty $\mathrm{R} \times$ Hr R & 18 & $31.6 \pm 1.19$ & $97.0 \pm 0.41$ & $99.2 \pm 0.45 \mathrm{a}$ & $71.4 \pm 1.32 \mathrm{~b}$ & $\mathrm{O}$ \\
\hline Hr R $\times$ Ty R & 16 & $33.6 \pm 1.50$ & $96.9 \pm 0.47$ & $97.6 \pm 0.55 \mathrm{a}$ & $71.2 \pm 1.76 \mathrm{~b}$ & $\mathrm{O}$ \\
\hline$\chi^{2}$-value & & $12.368 \mathrm{NS}$ & $8.482 \mathrm{NS}$ & $25.636^{* * *}$ & $109.245^{* * *}$ & \\
\hline $\operatorname{Ty} \times \operatorname{Tr}$ & 26 & $32.1 \pm 1.39 \mathrm{a}$ & $95.4 \pm 0.60 \mathrm{~b}$ & $97.0 \pm 0.61$ & $42.9 \pm 2.91 \mathrm{c}$ & $X$ \\
\hline $\operatorname{Tr} \times \operatorname{Ty}$ & 31 & $43.5 \pm 1.95 \mathrm{~b}$ & $94.4 \pm 0.69 \mathrm{ab}$ & $97.4 \pm 0.74$ & $11.5 \pm 1.13 \mathrm{a}$ & $X$ \\
\hline $\operatorname{Ty} \times \operatorname{Tr} R$ & 15 & $31.8 \pm 1.22 \mathrm{a}$ & $96.8 \pm 0.46 \mathrm{~b}$ & $99.0 \pm 0.48$ & $29.7 \pm 2.50 \mathrm{~b}$ & $X$ \\
\hline $\operatorname{Tr} \times$ Ty $R$ & 28 & $30.1 \pm 1.46 \mathrm{a}$ & $96.0 \pm 0.49 \mathrm{~b}$ & $98.4 \pm 0.55$ & $14.2 \pm 1.20 \mathrm{a}$ & $X$ \\
\hline Ty $\mathrm{R} \times \mathrm{Tr}$ & 20 & $35.9 \pm 1.56 \mathrm{ab}$ & $90.6 \pm 1.68 \mathrm{a}$ & $97.8 \pm 0.68$ & $16.8 \pm 2.67 \mathrm{a}$ & $X$ \\
\hline $\operatorname{Tr} \mathrm{R} \times \mathrm{Ty}$ & 17 & $32.3 \pm 1.56 \mathrm{a}$ & $96.7 \pm 0.42 \mathrm{~b}$ & $98.7 \pm 0.48$ & $13.8 \pm 1.18 \mathrm{a}$ & $x$ \\
\hline Ty $\mathrm{R} \times \operatorname{Tr} \mathrm{R}$ & 14 & $35.9 \pm 2.79 \mathrm{ab}$ & $96.7 \pm 0.89 b$ & $98.9 \pm 0.42$ & $30.1 \pm 2.60 \mathrm{~b}$ & $x$ \\
\hline $\operatorname{Tr} \mathrm{R} \times \mathrm{Ty} \mathrm{R}$ & 20 & $29.3 \pm 1.04 \mathrm{a}$ & $96.8 \pm 0.48 \mathrm{~b}$ & $98.3 \pm 0.60$ & $18.9 \pm 1.73 \mathrm{ab}$ & $x$ \\
\hline$\chi^{2}$-value & & $37.730^{* * *}$ & $21.329^{* *}$ & $9.236 \mathrm{NS}$ & $85.787^{* * *}$ & \\
\hline
\end{tabular}

${ }^{\mathrm{a} N u m b e r}$ of females tested.

${ }^{\mathrm{b}}$ Circles show compatible crosses and Xs incompatible ones.

cMeans differ significantly at $P<0.01$ (**) $^{*}$ and $P<0.001{ }^{* * *}$ (Kruskal-Wallis test); NS, not significant at $P>0.05$. Data are shown as mean \pm SE.

Values in a column followed by different letters are significantly different at $P<0.05$ (Scheffé's test).

Figure 2) was due to genetic differences, that is, nuclear genes.

\section{Crosses between the Wolbachia-infected Sd population and the Wolbachia-uninfected Sp and Os populations} As shown in Figure 2, the Sd population resulted in unidirectional incompatibility in crosses with the $\mathrm{Sp}$ or Os population (as shown by four diagonal cells). The crosses between females of either the $\mathrm{Sd}$ or $\mathrm{Sd} R$ population and males of either the Sp or Os population were compatible, but the reciprocal crosses were incompatible (Table 7). The incompatibility was shown by a reduced hatchability and/or a male-biased sex ratio. The crosses with the Sp population appeared to be more incompatible than the crosses with the Os population. This incompatibility closely resembled the incompatibility observed in Amphitetranychus quercivorus, which was due to a nuclear-cytoplasmic interaction (Gotoh et al (1995); in that paper, A. quercivorus was referred to as Tetranychus quercivorus).

\section{Discussion}

Using 25 P. mori populations, we performed 900 combinations $(30 \times 30)$ of crosses, which included 600 $(25 \times 25-25)$ interpopulation crosses $(=300(600 / 2)$ combinations of pairs of different populations (Figure 2)). The observed incompatibility in the crosses can be interpreted as the consequence of either nuclear-nuclear interactions, nuclear-cytoplasmic interactions or Wolbachia infection (see below). Bidirectional incompatibility caused by nuclear-nuclear interactions was observed in 99 combinations of interpopulations $(99 / 300=0.33$; shown by Xs enclosed in a double line excluding the two combinations of diagonal cells); five out of 10 combinations among the five Wolbachia-eliminated populations $((5 \times 5-5) / 2)$ (shown by hatching), 45 out of 190 combinations among the 20 Wolbachia-uninfected populations $((20 \times 20-20) / 2)$ (enclosed in a dotted line) and 49 out of 100 combinations between Wolbachia-eliminated and -uninfected populations $(5 \times 20$; enclosed in a dashed line in Figure 2). Although a few female progeny were produced in these crosses, nearly one-third of the combinations were incompatible, based on the nuclear background. This shows that there is genetic divergence among P. mori populations even when mites were collected from the same host plant, mulberry. We did not detect any geographical trends in the distribution of bidirectionally compatible populations. This is probably due to frequent transportation of mulberry trees, on which P. mori populations were collected, over the past 100 years as the sericulture industry was developed in Japan.

The incompatibility of the five Wolbachia-infected populations with the 25 different $P$. mori populations was complex. The $\mathrm{Sd}$ population did not show any Wolbachia-mediated incompatibility with the uninfected populations. The other four Wolbachia-infected populations ( $\mathrm{Hy}, \mathrm{Ty}, \mathrm{Hr}$ and $\mathrm{Tr}$ ) induced unidirectional incompatibility when their males were crossed with females of Wolbachia-uninfected populations. However, this Wolbachia-mediated incompatibility was not observed in all combinations of the populations between infected males and uninfected females. The Tr population, for example, was incompatible with three uninfected populations, whereas the Hy population was incompatible with 16 uninfected populations (Figure 2). This difference in the number of incompatible combinations may be due to different affinities of Wolbachia for 
Table 6 Compatibility of crosses between males of four Wolbachia-infected populations (Hy, Ty, Hr and Tr) and females of two Wolbachiauninfected populations ( $\mathrm{Hs}$ and $\mathrm{Tz}$ ) of P. mori

\begin{tabular}{|c|c|c|c|c|c|c|}
\hline Cross & $\mathrm{n}^{\mathrm{a}}$ & No. of eggs/female & Hatchability (\%) & Survival rate in immature stages (\%) & $\%$ Female offspring & Compatibility ${ }^{\mathrm{b}}$ \\
\hline \multicolumn{7}{|c|}{ Female $\times$ male } \\
\hline $\mathrm{Hs} \times \mathrm{Hy}$ & 16 & $28.6 \pm 1.33$ & $94.6 \pm 1.19$ & $96.5 \pm 0.73$ & $47.5 \pm 2.76$ & $x$ \\
\hline Hs $\times$ Hy R & 12 & $26.6 \pm 1.28$ & $97.3 \pm 0.61$ & $94.6 \pm 1.31$ & $66.5 \pm 1.57$ & $\mathrm{O}$ \\
\hline$Z^{c}$ & & $-0.933 \mathrm{NS}$ & $-2.062^{*}$ & $-1.011 \mathrm{NS}$ & $-4.295^{* * *}$ & \\
\hline $\mathrm{Tz} \times \mathrm{Hy}$ & 14 & $23.0 \pm 0.78$ & $95.1 \pm 1.44$ & $97.2 \pm 0.86$ & $37.7 \pm 3.22$ & $x$ \\
\hline Tz $\times$ Hy R & 15 & $22.9 \pm 0.71$ & $98.3 \pm 0.57$ & $95.9 \pm 1.18$ & $63.8 \pm 1.53$ & $\mathrm{O}$ \\
\hline Z & & $-0.000 \mathrm{NS}$ & $-1.831 \mathrm{NS}$ & $-0.549 \mathrm{NS}$ & $-4.587^{* * *}$ & \\
\hline $\mathrm{Hs} \times \mathrm{Ty}$ & 11 & $31.7 \pm 1.15$ & $66.3 \pm 4.47$ & $95.8 \pm 0.94$ & $65.5 \pm 2.80$ & $x$ \\
\hline Hs $\times$ Ty $R$ & 19 & $27.3 \pm 1.33$ & $96.0 \pm 0.75$ & $98.9 \pm 0.46$ & $76.5 \pm 1.33$ & $\mathrm{O}$ \\
\hline Z & & $-2.369^{*}$ & $-3.659^{* * *}$ & $-2.540^{*}$ & $-3.436^{* * *}$ & \\
\hline $\mathrm{Tz} \times \mathrm{Ty}$ & 17 & $33.3 \pm 0.61$ & $56.5 \pm 2.67$ & $97.2 \pm 0.77$ & $48.4 \pm 2.82$ & $x$ \\
\hline $\mathrm{Tz} \times \mathrm{Ty} R$ & 16 & $29.3 \pm 1.73$ & $95.9 \pm 0.63$ & $98.8 \pm 0.54$ & $78.6 \pm 1.35$ & $\mathrm{O}$ \\
\hline Z & & $-2.349^{*}$ & $-4.901^{* * *}$ & $-1.526 \mathrm{NS}$ & $-4.794^{* * *}$ & \\
\hline $\mathrm{Hs} \times \mathrm{Hr}$ & 17 & $30.9 \pm 1.31$ & $97.0 \pm 0.57$ & $99.3 \pm 0.42$ & $15.2 \pm 1.24$ & $X$ \\
\hline Hs $\times$ Hr R & 16 & $28.6 \pm 1.35$ & $97.9 \pm 0.59$ & $99.6 \pm 0.29$ & $76.3 \pm 1.20$ & $\mathrm{O}$ \\
\hline Z & & $-1.211 \mathrm{NS}$ & $-0.985 \mathrm{NS}$ & $-0.346 \mathrm{NS}$ & $-4.901^{* * *}$ & \\
\hline $\mathrm{Tz} \times \mathrm{Hr}$ & 21 & $30.2 \pm 1.22$ & $96.7 \pm 0.57$ & $98.6 \pm 0.39$ & $19.2 \pm 2.04$ & $X$ \\
\hline $\mathrm{Tz} \times \mathrm{Hr} \mathrm{R}$ & 19 & $29.5 \pm 1.27$ & $95.6 \pm 0.64$ & $98.8 \pm 0.42$ & $77.1 \pm 1.65$ & $\mathrm{O}$ \\
\hline Z & & $-0.354 \mathrm{NS}$ & -1.034 NS & $-0.302 \mathrm{NS}$ & $-5.405^{* * *}$ & \\
\hline $\mathrm{Hs} \times \mathrm{Tr}$ & 15 & $31.3 \pm 1.30$ & $52.9 \pm 2.09$ & $96.8 \pm 0.93$ & $53.2 \pm 2.27$ & $x$ \\
\hline Hs $\times \operatorname{Tr} R$ & 19 & $29.1 \pm 1.20$ & $97.0 \pm 0.53$ & $98.5 \pm 0.42$ & $70.8 \pm 1.21$ & $\mathrm{O}$ \\
\hline Z & & $-1.026 \mathrm{NS}$ & $-4.952^{* * *}$ & $-1.317 \mathrm{NS}$ & $-4.684^{* * *}$ & \\
\hline $\mathrm{Tz} \times \operatorname{Tr}$ & 16 & $33.3 \pm 0.64$ & $99.2 \pm 0.35$ & $98.5 \pm 0.47$ & $39.6 \pm 2.23$ & $x$ \\
\hline $\operatorname{Tz} \times \operatorname{Tr} R$ & 17 & $29.6 \pm 1.13$ & $96.2 \pm 0.43$ & $98.4 \pm 0.52$ & $77.7 \pm 1.04$ & $\mathrm{O}$ \\
\hline$Z$ & & $-2.386^{*}$ & $-3.999 * * *$ & $-0.200 \mathrm{NS}$ & $-4.901^{* * *}$ & \\
\hline
\end{tabular}

a Number of females tested.

${ }^{b}$ Circles show compatible crosses and Xs incompatible ones.

'Means differed significantly at $P<0.05{ }^{*}$ ) and $\left.P<0.001{ }^{* * *}\right)$ (Mann-Whitney $U$ test). NS: not significant at $P>0.05$.

Table 7 Compatibility of crosses between Wolbachia-infected Sd population and Wolbachia-uninfected Sp and Os populations of P. mori

\begin{tabular}{|c|c|c|c|c|c|c|}
\hline Cross & $\mathrm{n}^{\mathrm{a}}$ & No. of eggs/female & Hatchability (\%) & Survival rate in immature stages (\%) & $\%$ Female offspring & Compatibility $^{\mathrm{b}}$ \\
\hline \multicolumn{7}{|c|}{ Female $\times$ male } \\
\hline $\mathrm{Sd} \times \mathrm{Sp}$ & 12 & $34.7 \pm 0.54 \mathrm{c}$ & $99.5 \pm 0.35 b$ & $99.3 \pm 0.39$ & $63.9 \pm 1.27 \mathrm{cde}$ & $\mathrm{O}$ \\
\hline $\mathrm{Sd} \times \mathrm{Os}$ & 18 & $32.7 \pm 0.86 \mathrm{bc}$ & $99.5 \pm 0.27 b$ & $97.8 \pm 0.41$ & $73.8 \pm 0.59 \mathrm{e}$ & $\mathrm{O}$ \\
\hline $\mathrm{Sd} R \times S p$ & 15 & $27.9 \pm 1.67 \mathrm{ab}$ & $97.8 \pm 0.61 \mathrm{~b}$ & $97.0 \pm 0.85$ & $64.3 \pm 1.70 \mathrm{cde}$ & $\mathrm{O}$ \\
\hline $\mathrm{Sd} \mathrm{R} \times \mathrm{Os}$ & 12 & $23.9 \pm 0.96 \mathrm{a}$ & $97.6 \pm 0.62 \mathrm{~b}$ & $95.1 \pm 1.16$ & $67.4 \pm 1.99 \mathrm{de}$ & $\mathrm{O}$ \\
\hline $\mathrm{Sp} \times \mathrm{Sd}$ & 38 & $34.9 \pm 0.87 \mathrm{c}$ & $45.0 \pm 2.53 \mathrm{a}$ & $94.3 \pm 1.03$ & $7.9 \pm 2.97$ a & $X$ \\
\hline $\mathrm{Sp} \times \mathrm{SdR}$ & 11 & $24.3 \pm 0.85 \mathrm{a}$ & $71.9 \pm 3.52 \mathrm{a}$ & $95.3 \pm 1.33$ & $25.4 \pm 2.71 \mathrm{~b}$ & $X$ \\
\hline Os $\times \mathrm{Sd}$ & 15 & $35.0 \pm 1.37 \mathrm{c}$ & $99.0 \pm 0.38 \mathrm{~b}$ & $97.0 \pm 0.84$ & $51.3 \pm 1.05 c$ & $X$ \\
\hline Os $\times$ Sd R & 13 & $23.2 \pm 0.81 \mathrm{a}$ & $97.9 \pm 0.71 \mathrm{~b}$ & $96.5 \pm 0.92$ & $51.7 \pm 2.12 \mathrm{~cd}$ & $x$ \\
\hline$\chi^{2}$-value & & $73.544^{* * *}$ & $106.117^{* * *}$ & $12.966 \mathrm{NS}$ & $115.745^{* * *}$ & \\
\hline
\end{tabular}

aNumber of females tested.

${ }^{\mathrm{b} C}$ ircles show compatible crosses and Xs incompatible ones.

'Means differ significantly at $P<0.001{ }^{(* * *}$ (Kruskal-Wallis test); NS, not significant at $P>0.05$. Data are shown as mean \pm SE. Values in a column followed by different letters are significantly different at $P<0.05$ (Scheffé's test). 
the nuclear genes of the uninfected populations; it suggests the presence of 'rescue genes' in the host genome.

The compatibilities among the five Wolbachia-infected populations were relatively complicated, apparently because nuclear genes of these populations also affected their compatibilities. Bidirectional compatibility was observed in crosses between $\mathrm{Sd}$ and Hy, and between Hy and Ty. The Wolbachia strain of the Hy and Ty populations was identical as far as compatibility and wsp sequence are concerned (Gotoh et al, 2003). Crosses between $\mathrm{Sd}$ and Ty were bidirectionally incompatible, although their wsp sequences are identical. This suggests that the host genotype may influence the Wolbachia effect. Thus, even though Wolbachia have identical wsp sequences (Gotoh et al, 2003), they may interact differently with different host genotypes, resulting in different crossability patterns, as has been reported in butterflies (Jiggins et al, 2002). The contradictory results in compatibility of the $\mathrm{Sd}$ population are discussed below. In contrast, crosses between Sd and Ty, between Ty and $\mathrm{Hr}$ and between $\mathrm{Hr}$ and $\mathrm{Tr}$ became reciprocally compatible after the antibiotic treatment, indicating that the incompatibility in these populations was due to infection by a different strain of Wolbachia.

The Sd population was bidirectionally compatible with the Hy population as well as with the 11 Wolbachiauninfected populations, but was bidirectionally incompatible with the Ty population. Furthermore, the Sd males were compatible with both Sd R and Hy R females. These results can be summarized as follows: Wolbachia of the Sd population behave as a modification-negative strain to the Hy population and 11 Wolbachia-uninfected populations, whereas the bacteria behave as a modification-positive strain only to the Ty population. The incompatibility observed in crosses between the Sd and Ty populations may be due to different intensities of the modification function included in males through an affinity between genotypes. The wsp gene sequences appear to rule out the possibility of double infection of Wolbachia against the Sd and Ty populations (Gotoh et al, 2003). We also failed to detect CFB (CLO) in these populations. CFB are also maternally transmitted bacteria that cause a diverse array of reproductive manipulations, including male killing, parthenogenesis, feminization and CI in insects and mites (Hurst et al, 1999; Weeks et al, 2001; Zchori-Fein et al, 2001; Hunter et al, 2003).

Incompatibility based on the nuclear-cytoplasmic interaction was observed between $S p$ and $S d$ and between Os and Sd (Table 7). The southern Sd population showed unidirectional incompatibility when crossed with the northern Sp or Os populations; the Sp or Os females were incompatible with the Wolbachia-eliminated $\mathrm{Sd}$ males (Sd R males), but the reciprocal crosses were compatible (Table 7). The Sd population was infected with Wolbachia but this unidirectional incompatibility was not due to Wolbachia. A similar result was obtained in crosses between the Tsukuba $\left(36^{\circ} \mathrm{N}\right)$ and Sapporo $\left(43^{\circ} \mathrm{N}\right)$ populations of $A$. quercivorus (Gotoh et al, 1995). In $A$. quercivorus, the Sapporo females were incompatible with the Tsukuba males, which resulted in a low egg hatchability and a male-biased sex ratio, whereas the reciprocal crosses were compatible and produced normal progenies with a female-biased sex ratio. This unidirec- tional incompatibility is considered to be due to the negative interaction between the nucleus of the Tsukuba males and the cytoplasm of the Sapporo females (Gotoh et al, 1995). For P. mori, the northern Sp and Os females were incompatible with the southern Sd and Sd R males, but the reciprocal crosses were compatible (Table 7). Although the hatchability and the sex ratio observed in the incompatible crosses were higher in $P$. mori than those in A. quercivorus, the nuclear-cytoplasmic interactions appear to be responsible for the unidirectional incompatibility between Sd and Sp (Os) populations.

Similar complicated crossing types in a species have mainly been reported in Culex pipiens (Laven, 1967; Magnin et al, 1987) and Drosophila simulans (Mercot et al, 1995; Rousset and Solignac, 1995), although the latter has been well worked out. Crossing experiments between European, African and American populations of Culex pipiens disclosed all types of compatibility: bidirectional compatibility, unidirectional incompatibility and bidirectional incompatibility in the crosses with other populations (Laven, 1959, 1967). A highly complicated incompatibility pattern was observed in P. mori along with the combination of nuclear-nuclear and nuclearcytoplasmic interactions. The complexity of CI observed in some insect species makes it difficult to understand the mechanisms of CI clearly. Future individual approaches to the study of nuclear-nuclear incompatibility, nuclear-cytoplasmic incompatibility and CI should gradually unravel the complexity and contribute for elucidation of the mechanisms.

\section{Acknowledgements}

We thank Koh Gomi, Yasuki Kitashima and Sawako Kawai for their assistance. This work was supported in part by Grants-in-Aid for Scientific Research (nos. 09660040, 12460019 and 15380040) from the Ministry of Education, Science, Sports and Culture of Japan.

\section{References}

Bourtzis K, Dobson SL, Braig HR, O'Neill SL (1998). Rescuing Wolbachia have been overlooked.... Nature 391: 852-853.

Breeuwer JAJ (1997). Wolbachia and cytoplasmic incompatibility in the spider mites Tetranychus urticae and T. turkestani. Heredity 79: 41-47.

Breeuwer JAJ, Werren JH (1990). Microorganisms associated with chromosome destruction and reproductive isolation between two insect species. Nature 346: 558-560.

Gomi K, Gotoh T, Noda H (1997). Wolbachia having no effect on reproductive incompatibility in Tetranychus kanzawai Kishida (Acari: Tetranychidae). Appl Entomol Zool 32: 485-490.

Gotoh T, Gomi K, Nagata T (1999a). Incompatibility and host plant differences among populations of Tetranychus kanzawai Kishida (Acari: Tetranychidae). Appl Entomol Zool 34: 551-561.

Gotoh T, Higo Y (1997). Differences in host range and reproductive compatibility among populations of Panonychus mori Yokoyama (Acari: Tetranychidae). Int J Acarol 23: 119-125.

Gotoh T, Noda H, Hong X-Y (2003). Wolbachia distribution and cytoplasmic incompatibility based on a survey of 42 spider mite species (Acari: Tetranychidae) in Japan. Heredity 91 208-216.

Gotoh T, Oku H, Moriya K, Odawara M (1995). Nucleuscytoplasm interactions causing reproductive incompatibility 
between two populations of Tetranychus quercivorus Ehara et Gotoh (Acari: Tetranychidae). Heredity 74: 405-414.

Gotoh T, Sugasawa J, Nagata T (1999b). Reproductive compatibility of the two-spotted spider mite (Tetranychus urticae) infected with Wolbachia. Entomol Sci 2: 289-295.

Hoffmann AA, Turelli M, Simmons GM (1986). Unidirectional incompatibility between populations of Drosophila simulans. Evolution 40: 692-701.

Holden PR, Brookfield JFY, Jones P (1993). Cloning and characterization of an ftsZ homologue from a bacterial symbiont of Drosophila melanogaster. Mol Gen Genet $\mathbf{2 4 0}$ 213-220.

Hunter MS, Perlman SJ, Kelly SE (2003). A bacterial symbiont in the Bacteroidetes induces cytoplasmic incompatibility in the parasitoid wasp Encarsia pergandiella. Proc R Soc Lond B 270: 2185-2190.

Hurst GDD, Bandi C, Sacchi L, Cochrane AG, Bertrand D, Karaca I et al (1999). Adonia variegata (Coleoptera: Coccinellidae) bears maternally inherited Flavobacteria that kill males only. Parasitology 118: 125-134.

Jiggins FM, Bentley JK, Majerus MEN, Hurst GDD (2002). Recent changes in phenotype and patterns of host specialization in Wolbachia bacteria. Mol Ecol 11: 1275-1283.

Kurtti TJ, Munderloh UG, Andreadis TG, Magnarelli LA (1996). Tick cell culture isolation of an intracellular prokaryote from the tick Ixodes scapularis. J Invertebr Pathol 67: 318-321.

Laven H (1959). Speciation by cytoplasmic isolation in the Culex pipiens-complex. Cold Spring Harbor Symp 24: 166-173.

Laven H (1967). Speciation and evolution in Culex pipiens. In: Wright JW, Pal R (eds). Genetics of Insect Vectors of Disease. Elsevier: Amsterdam. pp 251-275.

Magnin M, Pasteur N, Raymond M (1987). Multiple incompatibilities within populations of Culex pipiens L in Southern France. Genetica 74: 125-130.

Mercot H, Llorente B, Jacques M, Atlan A, Montchamp-Moreau C (1995). Variability within the Seychelles cytoplasmic incompatibility system in Drosophila simulans. Genetics 141: 1015-1023.
Mercot H, Poinsot D (1998). ... and discovered on Mount Kilimanjaro. Nature 391: 853.

Noda H, Koizumi Y, Zhang Q, Deng K (2001). Infection density of Wolbachia and incompatibility level in two planthopper species, Laodelphax striatellus and Sogatella furcifera. Insect Biochem Mol Biol 31: 727-737.

O'Neill SL (1989). Cytoplasmic symbionts in Tribolium confusum. Invertebr Pathol 53: 132-134.

O'Neill SL, Giordano R, Colbert AME, Karr TL, Robertson HM (1992). 16S rRNA phylogenetic analysis of the bacterial endosymbionts associated with cytoplasmic incompatibility in insects. Proc Natl Acad Sci USA 89: 2699-2702.

O'Neill SL, Hoffmann AA, Werren JH (eds) (1997). Influential Passengers: Inherited Microorganisms and Arthropod Reproduction. Oxford University Press: New York. 214pp.

Rousset F, Solignac M (1995). Evolution of single and double Wolbachia symbioses during speciation in the Drosophila simulans complex. Proc Natl Acad Sci USA 92: 6389-6393.

Stouthamer R, Luck RF, Hamilton WD (1990). Antibiotics cause parthenogenetic Trichogramma (Hymenoptera/Trichogrammatidae) to revert to sex. Proc Natl Acad Sci USA 87: 2424-2427.

Weeks AR, Marec F, Breeuwer JAJ (2001). A mite species that consists entirely of haploid females. Science 292: 2479-2482.

Weeks AR, Velten R, Stouthamer R (2003). Incidence of a new sex-ratio-distorting endosymbiotic bacterium among arthropods. Proc R Soc Lond Ser B 270: 1857-1865.

Werren JH, O'Neill SL (1997). The evolution of heritable symbionts. In: O'Neill SL, Hoffmann AA, Werren JH (eds) Influential Passengers: Inherited Microorganisms and Arthropod Reproduction. Oxford University Press: New York. pp 1-41.

Zchori-Fein E, Gottlieb Y, Kelly SE, Brown JK, Wilson JM, Karr TL et al (2001). A newly discovered bacterium associated with parthenogenesis and a change in host selection behavior in parasitoid wasps. Proc Natl Acad Sci USA 98: 12555-12560.

Supplementary Information accompanies the paper on Heredity website (http://www.nature.com/hdy) 